Cinémas

Revue d'études cinématographiques

Journal of Film Studies

\title{
De l'" automate spirituel " ou le temps dans le cinéma moderne selon Gilles Deleuze
}

\section{Réda Bensmaïa}

Volume 5, numéro 1-2, automne 1994

Le Temps au cinéma

URI : https://id.erudit.org/iderudit/1001012ar

DOI : https://doi.org/10.7202/1001012ar

Aller au sommaire du numéro

Éditeur(s)

Cinémas

ISSN

1181-6945 (imprimé)

1705-6500 (numérique)

Découvrir la revue

Citer cet article

Bensmaïa, R. (1994). De l'« automate spirituel » ou le temps dans le cinéma moderne selon Gilles Deleuze. Cinémas, 5(1-2), 167-186.

https://doi.org/10.7202/1001012ar
Résumé de l'article

Dans ce texte, l'auteur analyse le concept d'« automate spirituel » tel qu'il est mis en oeuvre dans les deux livres que le philosophe Gilles Deleuze a consacrés au cinéma : L'Image-Mouvement et L'Image-Temps. Après une brève analyse du statut des concepts dans la philosophie de Deleuze, l'auteur montre, dans une première étape, l'importance du concept d'« automate spirituel » dans son oeuvre et en retrace l'origine en remontant à des auteurs aussi différents qu'Élie Faure, S. M. Eisenstein, Antonin Artaud, Heidegger, Rilke, etc. Dans un deuxième temps, il tente de montrer de quelle manière Gilles Deleuze " intègre " ce concept à son oeuvre en le convertissant en ce qu'il appelle un « transformateur ». Le reste de l'article s'attache à montrer à partir d'exemples précis de quelle manière ce concept « rigoureux et inexact » bien deleuzien réagit sur les principaux concepts du cinéma (cadrage, montage, hors-champ, plan d'ensemble, etc.) et vient déterminer de manière essentielle la réflexion sur le temps au cinéma. 


\section{De l'«automate spirituel» ou le temps dans le cinéma moderne selon Gilles Deleuze}

\section{Réda Bensmaïa}

\section{RÉSUMÉ}

Dans ce texte, l'auteur analyse le concept d'"automate spirituel " tel qu'il est mis en œuvre dans les deux livres que le philosophe Gilles Deleuze a consacrés au cinéma: L'Image-Mouvement et L'Image-Temps. Après une brève analyse du statut des concepts dans la philosophie de Deleuze, l'auteur montre, dans une première étape, l'importance du concept d' "automate spirituel" dans son œuvre et en retrace l'origine en remontant à des auteurs aussi différents qu'Élie Faure, S. M. Eisenstein, Antonin Artaud, Heidegger, Rilke, etc. Dans un deuxième temps, il tente de montrer de quelle manière Gilles Deleuze "intègre" ce concept à son œuvre en le convertissant en ce qu'il appelle un "transformateur". Le reste de l'article s'attache à montrer à partir d'exemples précis de quelle manière ce concept « rigoureux et inexact " bien deleuzien réagit sur les principaux concepts du cinéma (cadrage, montage, hors-champ, plan d'ensemble, etc.) et vient déterminer de manière essentielle la réflexion sur le temps au cinéma.

\section{ABSTRACT}

In this text, the author analyses the concept of "spiritual automaton" as it is used in the two books that philosopher Gilles Deleuze devoted to film: L'ImageMouvement and L'Image-Temps. After a brief examination of the status of the concepts in Deleuze's philosophy, the author points, first of all, to the importance of the concept of spiritual automaton in his work and tra- 
ces its origins back to the work of writers as diverse as Élie Faure, S.M. Eisenstein, Antonin Artaud, Heidegger, Rilke, etc. Secondly, he tries to show how Deleuze "integrates" this concept into his work by converting it into what he calls a "transformer." The remainder of the article seeks to show, using concrete examples, how this "rigorous and inexact" (and very Deleuzian) concept interacts with the primary concepts of cinema (framing, editing, off-camera, long shot, etc.) and determines in an essential way the reflection on time in cinema.

Le "fil» conducteur de ce texte est celui d' "automate spirituel ", soit un "concept " qui n'intervient que relativement tard dans les livres que Gilles Deleuze a consacrés au cinéma ${ }^{1}$, mais qui, ainsi que j'essaierai de le montrer peu à peu, n'en joue pas moins un rôle essentiel dans l'économie générale de la conception deleuzienne du temps au cinéma.

Ce qu'une certaine familiarité avec le travail de Deleuze permet de bien mettre en évidence, c'est, d'une part, que les concepts qu'il crée n'ont point besoin de figurer dans le titre d'un livre ou d'un chapitre pour jouer un rôle important dans son analyse et, d'autre part, que ces mêmes concepts doivent toujours être pris en considération en fonction de la problématique théorique dans laquelle ils sont repris et révalués. C'est dire qu'à de très rares exceptions près, aucun des concepts deleuziens ne peut généralement être pris tel quel ou plutôt à la manière dont telle ou telle "tradition" philosophique l'aura légué à la postérité. De nombreux critiques ont su relever l'originalité avec laquelle Deleuze pratique l'histoire de la philosophie et la très grande versatilité de l'utilisation qu'il fait de ses concepts "canoniques". En consacrant, coup sur coup, deux livres au cinéma, Gilles Deleuze n'a encore une fois pas dérogé à la règle qui consiste chez lui à arracher les concepts à leurs contextes théoriques d'origine, pour les réactiver, en réévaluer la teneur et leur faire jouer un nouveau rôle, en un mot les transformer en "personnages conceptuels ${ }^{2}$ ». Ainsi, pour Gilles Deleuze, les concepts philosophiques ne sont jamais des entités préformées une fois pour toutes, mais toujours matière à perlaboration et connexion, mise 
en crise et invention nouvelles. L' "automate spirituel" est, en ce sens, l'un des plus beaux exemples de ces "personnages" philosophico-conceptuels que la théorie du cinéma ait jamais inventés pour rendre compte de son "objet".

On trouve un bon exemple du mouvement de pensée qui anime la création de ces derniers avec la notion de "Corps sans organes" $(\mathrm{CsO})$. Une notion, rappelons-le, que Deleuze avait d'abord introduite pour la première fois dans Logique du sens et présentée comme ayant été empruntée à Antonin Artaud (un poète) et à Gisela Pankov (une psychiatre). Quelques années plus tard, alors qu'il travaille avec Félix Guattari, Deleuze reprendra cette notion pour lui faire jouer un rôle essentiel, dans L'Anti-CEdipe cette fois. Mais ce qu'il est important de signaler c'est que, du fait de ce transfert, ce concept changera totalement de nature et de définition: le $\mathrm{CsO}$ ne tirera plus seulement sa valence et son efficace de l'œuvre des auteurs qui avaient permis de l'intuitionner originairement, mais d'au moins deux nouveaux systèmes de pensée, qui renvoient eux-mêmes à deux nouveaux régimes théoriques. En effet, comme l'a bien montré André Pierre Colombat dans le bel article "A Thousand Trails : to Work with Deleuze ", consacré au "travail " des concepts dans Mille Plateaux, de concept articulé autour de l'idée de non-sens telle qu'elle avait été développée par Antonin Artaud et par Lewis Caroll, le $\mathrm{CsO}$ sera donné à présent à la fois "comme un œuf" (soit comme une métaphore biologique) et comme une "substance spinoziste" (soit, cette fois, comme un concept proprement philosophique)! Immanquablement, la juxtaposition de ces deux champs, apparemment "incompatibles" d'explication créera du non-sens ou, si l'on préfere, un "excès de sens" qui mettra l'intellect et l'imagination du lecteur en mouvement: "Le concept de "Corps sans Organes" sera dès lors défini entre deux séries hétérogènes du fait d'un va-et-vient infini entre la conception deleuzienne de l'œuf qu'il emprunte à François Dalcq et sa conception de la substance spinoziste" (Colombat, p. 14), qu'il tire cette fois de sa propre interprétation de L'Éthique de Spinoza ${ }^{3}$. Dans Mille Plateaux, le $\mathrm{CsO}$ réémergera pour jouer un rôle encore plus complexe ou plus exactement en (se) compliquant - au sens rhétorique - transversalement de 
nouvelles déterminations : cette fois, le $\mathrm{CsO}$ se "souvient" d'avoir été un œuf ainsi qu'une substance spinoziste, mais se révèle être aussi bien "matière intensive et informelle" (Colombat, p. 14), L'Éthique de Spinoza, "plan de consistance du désir ", Héliogabale, Logos Spermaticos, et bien d'autres choses encore ${ }^{4}$ ! Comme "concept rigoureux et inexact", le $\mathrm{CsO}$ sera en constante métamorphose, "[...] occupant l'espace intermédiat qui permet aux intensités et aux flux désirants de circuler avant de s'actualiser dans différentes formes de pensées ou dans des organismes vivants, sur un plan physique ou métaphysique" (Colombat, p. 14).

Avec le concept d' "automate spirituel ", nous assistons, à propos du cinéma, à un mouvement de pensée du même type. Même s'il est tiré initialement du Traité de la réforme de l'entendement de Spinoza (p. 73-74), l' «automate spirituel» sera vite transformé en un "concept" du type Jabberwocky, Snark ou $\mathrm{CsO}$. C'est dire que nous aurons encore affaire à l'un de ces concepts "inexacts et rigoureux" qui traversent l'œuvre de Deleuze et qui font la richesse des effets théoriques et pratiques qu'il en tire.

C'est d'abord un concept "inexact " parce qu'il est difficile, sinon impossible, de lui assigner une "origine" unique et stable. En effet, Deleuze n'hésite pas à en rapporter l'usage et l'efficace à des écrivains, artistes, historiens, créateurs et théoriciens aussi différents qu'Élie Faure, Jean Epstein, S. M. Eisenstein pour démarrer, mais c'est pour aussitôt leur associer et leur connecter de manière complexe et contradictoire un écrivain comme Antonin Artaud et des cinéastes comme Pier Paolo Pasolini ou Jean-Luc Godard, et finir (pour reprendre, comme à nouveaux frais la question) avec un poète comme Paul Valéry ou des philosophes comme Spinoza ou Heidegger.

Mais de plus, le concept d' "automate spirituel" peut cependant être, en même temps, dit "rigoureux", dans le sens que j'ai suggéré plus haut, en ce que manifestement le "transfert" et la "traduction" auxquels il est soumis, eux, ne sont ni gratuits ni improvisés: ils obéissent au contraire à une démarche de pensée des plus consistantes. Selon quel critère peut-on juger de cette consistance? Comment peut-on dire d'un concept qu'il est à la 
fois " inexact " et "rigoureux " ? Pour quelles raisons cette recréation ou réactivation d'un concept à l'occasion d'une réflexion sur le cinéma? Enfin: de quelle manière ce "concept" affectet-il le temps au cinéma?

Ce qui intéresse Deleuze dans le concept spinoziste d' "automate spirituel ", ce n'est point tant l'aspect "automatique" ou "mécanique" - Spinoza n'est du reste pas du tout un philosophe "mécaniste" —, mais ce qui en lui ou à partir de lui renvoie à une "méthode" qui emprunte son point de départ à la géométrie, mais ne trouve cependant sa forme intrinsèque d'action que dans le mouvement de pensée qui lui fait dépasser ses propres limites ordinaires et s'élever à l'Essence comme "raison génétique de toutes les propriétés connaissables" (Deleuze, 1968, p. 75-76). Chez Deleuze, l'intérêt de l'"automate spirituel" spinoziste tient à au moins deux facteurs indissociables: l'immanence de la pensée et la théorie du parallélisme. "Automate spirituel " signifie d'abord, écrit Deleuze, "[...] qu’une idée, étant un mode de pensée, ne trouve pas sa cause (efficiente et formelle) ailleurs que dans l'attribut pensée. De même, un objet quel qu'il soit ne trouve[ra] sa cause efficiente et formelle que dans l'attribut dont il est le mode et dont il enveloppe le concept " (Deleuze, 1968, p. 101).

Ces définitions ont l'air bien "abstraites" ainsi reformulées hors contexte, mais elles deviennent beaucoup plus efficaces et concrètes dès que l'on comprend que ce qu'elles impliquaient pour Spinoza n'était autre chose que la puissance du parallélisme: car dire qu'une idée ou qu'un objet ne trouvent leur cause efficiente et formelle "que dans l'attribut dont il sont le mode", c'est affirmer que "toute causalité efficiente ou formelle (et à plus forte raison matérielle et finale) est exclue entre les idées et les choses, les choses et les idées". C'est dire que ce qui retient l'attention de Deleuze dans l' " automatisme " de Spinoza, c'est la conséquence qu'il tire de ses axiomes de départ: soit l'affirmation de l'indépendance des deux séries, série des choses et série des idées; et bientôt, pour Deleuze, indépendance de la série d'images et de la série des objets, des choses, ou des corps; série de mots et d'images, etc. Et surtout "autonomie" et "automatisme" de la pensées. 
Comme la méthode de Spinoza, la méthode de Deleuze dans Cinéma I. L'Image-mouvement et Cinéma II. L'Image-temps comportera, elle aussi, trois grands moments (au moins), chacun étroitement impliqué par les autres. 1) Un premier moment où l'appréhension du mouvement et des "objets" qu'il entraîne est livrée à l'intuition ou aux "données immédiates de la conscience", et c'est le moment de l'analyse du cinéma comme simple "image-mouvement" ou, si l'on préfère, "imagereprésentation ${ }^{6} " ; 2$ ) un moment où ce n'est plus le mouvement en tant que tel qui préside à l'analyse, mais sa dislocation, et c'est alors l'irruption de l'image-temps et de l'«image-figure "; enfin, 3) un troisième mouvement, de synthèse, mais disjonctive, où ce sera à un Dehors "impensable " que s'ordonnera... la pensée. Pensée de l'impensable: "automate spirituel ".

Tel est, très schématiquement exposé, le "programme " qui est ordonné à partir de la réactivation du concept d' "automate spirituel ". Mais c'est à partir de cette dernière que tout commence, car comme je l'ai suggéré plus haut, en convoquant ce concept, il n'a jamais été question pour Deleuze de l'«appliquer" tel quel au cinéma, mais tout au contraire de se servir du cinéma pour transformer un concept "exact" - trop "exact " chez Spinoza - en un opérateur d'analyse ou en accélérateur de concepts, soit en un concept "inexact et rigoureux " qui lui permettra de "lever en nous" la nouvelle pensée du cinéma. Autrement dit, l'" automate spirituel " comme "transformateur" de régime des concepts et des images du cinéma. Comme l'écrit Deleuze, "[...] le mouvement automatique [du cinéma] fait lever en nous un automate spirituel, qui réagit à son tour sur lui" (1985, p. 203). Et de fait, à partir du moment où il sera confronté à la logique disjonctive qui est à l'œuvre au cinéma, le concept d'" automate spirituel" ne désignera plus, comme dans la philosophie classique, "La [simple] possibilité logique ou abstraite de déduire formellement les pensées les unes des autres, mais le circuit dans lequel elles entrent avec l'image-mouvement, la puissance commune de ce qui force à penser et de ce qui pense sous le choc: un noochoc" (p. 204).

Comme on le voit, l'effet du déplacement de l'"automate", d'un champ théorique donné à un autre, est à double détente: 
en même temps qu'il arrache la philosophie spinoziste - et peutêtre toute la philosophie - à son "formalisme" latent, il arrache le cinéma à son "mécanisme" patent (le simple "choc") pour y révéler ce qui nous force à penser (par-delà et en deçà de l'image-mouvement et de l'image-action). Dès que l'on comprend la nature de l'intervention de l' "automate spirituel" au cinéma, "Tout se passe comme si le cinéma nous disait: avec moi, avec l'image-mouvement, vous ne pouvez pas échapper au choc qui éveille le penseur en vous. Un automate subjectif et collectif pour un mouvement automatique: l'art des "masses" " (p. 204).

Tant qu'ils n'avaient à leur disposition qu'une conception formelle du mouvement et de la production des idées au cinéma idées encore représentatives! - les cinéastes et les théoriciens du cinéma ne pouvaient qu'en rater la dimension noétique. Mais c'est qu'ils confondaient le "choc" et le "noochoc" et réduisaient par là même les "vibrations" de l'image-mouvement à la "violence figurative du représenté". Une "réduction" doublée d'une confusion, qui ne tarda pas à assimiler de manière indue le cinéma comme "automate spirituel" avec le cinéma comme "art de propagande" ou de reproduction de la réalité! Selon Deleuze, malgré sa vigilance théorique, même un cinéaste aussi génial que S. M. Eisenstein s'est laissé prendre à une conception encore toute "mécanique" de la dimension noétique de l' "automatisme " cinématographique.

En effet, si Eisenstein avait bien repéré le "mouvement" qui, dans le cinéma, va du choc de deux images à la pensée, ou, comme il le disait lui-même, au "concept"; s'il avait bien mis en évidence la dialectique retorse qui renverse ce premier "mouvement " pour passer du "concept " à l' "affect" et de l'"organique" au "pathétique", il n'est jamais parvenu à produire une idée adéquate de la nature de l' "automatisme" qui était à l'œuvre au cinéma. Comme le dit bien Deleuze, avec Eisenstein: " $[\ldots]$ on ne va plus de l'image-mouvement à la pensée claire du tout qu'elle exprime, on va d'une pensée du tout présupposée, obscure, aux images agitées, brassées qui l'expriment ${ }^{7}$ " (1985, p. 207). Et en ce sens, Eisenstein serait bien parvenu à intuitionner la nature complexe du "choc" cinématographique 
"sous la figure de l'opposition" et la pensée qu'il engage "sous forme de l'opposition surmontée ou la transformation des opposés" (p. 206). Mais pour être "dialectique", cette transformation n'a pas renoncé pour autant à l'idée d'une totalité comme harmonique des parties ("plans") qui leur préexiste dans un Sujet ou un Monde. S'il indique bien un passage (dialectique) d'un Logos qui "unifie les parties" à un Pathos qui «les baigne et se répand en elles" (p. 207), le choc en retour qui va du concept inconscient (du tout) à l'image-matière — " jets de crème, fontaines d'eau lumineuses, feux jaillissants, zigzags formant des chiffres" de La ligne générale, par exemple — Eisenstein croit encore au cinéma comme un "appareil" qui permet de revenir aux images et de les enchaîner suivant les exigences d'un "monologue intérieur" qu'il réduit encore "au cours de la pensée d'un homme" (p. 207).

Pour s'être débarrassé d'une conception du cinéma qui avait tendance à rabattre la dimension rhétorique qui le détermine sur les dimensions logique et grammaticale, Eisenstein n'aurait pas mesuré la portée plus purement noétique du cinéma: avec lui, le cinéma se serait éveillé à la puissance de l'« image-figure», c'est-àdire au jeu formel "[...] qui [au cinéma] donne à l'image une charge affective qui vient redoubler le choc sensoriel " (Deleuze, 1985 , p. 207), mais il n'aurait pas pris la mesure de ce qui, au cinéma encore, peut renvoyer à un "dehors" qui n'est réductible ni à une "intériorité" comme subjectivité ni à une "extériorité" comme res extensa ou monde "extérieur". Il faudra donc envisager un troisième "moment" qui ne consistera plus à aller de l'image à la pensée (soit du percept au concept) ou du concept à l'affect (soit, de la pensée à l'image), mais à dépasser cette dialectique pour créer un mouvement où le concept et l'image ne feront plus qu'un: «[...] le concept est en soi dans l'image, l'image est pour soi dans le concept. Ce n'est pas plus l'organique et le pathétique, mais le dramatique, le pragmatique, la praxis ou la pensée-action " (p. 210). Et en ce sens, pour Deleuze, Eisenstein est bien le cinéaste-théoricien qui aurait arraché le cinéma aux impératifs du cinéma "narratif-représentatif» et qui, de ce fait, l'aurait engagé dans la voie d'une noétique cinématographique, mais tout se passe comme s'il l'avait trop vite réduit à un "auto- 
matisme" - soit à un instrument au service de la révolution historique ou de la raison dialectique. Avec S. M. Eisenstein, l' "automate spirituel" est devenu l' "Homme révolutionnaire", comme avec Léni Riefenstahl, il deviendra (l'instrument de) l'"Homme fasciste". Dans les deux cas, la "bataille audiovisuelle" a abouti à une croyance qui a lâché la proie pour l'ombre: elle est devenue malgré tout un puissant instrument de capture du monde extérieur — " notre monde " ! - au détriment des forces originaires qu'elle tirait sans le savoir d'un "Dehors non formable et non formé ${ }^{10}$ ".

Et c'est à ce niveau que ce que nous appellerons volontiers le "Transformateur-Automate Spirituel " " (T.-A.S.) montre toute son efficacité, car au lieu que ce dernier constat aboutisse à une impasse, c'est plutôt à une relance des problèmes qu'il participe. En effet, ce que Deleuze peut mettre en évidence grâce à ce véritable "transformateur" de concepts qu'est l'" automate spirituel", c'est que l'échec du cinéma de l'image-mouvement et de l'image-action et la médiocrité crasse qui guette ses productions les plus commerciales ne peuvent être attribués ni à la pauvreté des idéologies ni au "fascisme de la production" (1985, p. 214215) — après tout, Riefenstahl a produit de "beaux " films! —, mais avant tout à une pensée qui n'a pas su toujours s'élever à la hauteur de la puissance intrinsèque de l' "automatisme" cinématographique. Tout se passe pour Deleuze comme si le cinéma avait échoué à rendre compte et à jouer à plein de ses potentialités non par excès d' "automatisme", mais par manque de radicalisme. Et c'est à ce niveau que l'œuvre d'un écrivain comme Antonin Artaud jouera un rôle des plus importants.

Avec Antonin Artaud, le "T.-A.S." se chargera de nouvelles valences et permettra de penser à nouveaux frais l'économie des concepts clés de montage et de tout au cinéma. En effet, n'étant plus conçu, cette fois, comme "[...] la [simple] possibilité logique d'une pensée qui déduirait formellement ses idées les unes des autres" (exemple: le "montage-roi» eisensteinien), et encore moins comme " $[\ldots]$ la puissance physique d'une pensée qu'on monterait en circuit avec l'image automatique" (Deleuze, 1985, p. 217) (exemple: l' "image-choc" expressionniste), mais comme "momie ${ }^{12}$ ", le "T.-A.S." ne servira plus de caution aux

De l'“automate spirituel » ou le temps dans le cinéma moderne selon Gilles Deleuze 
entreprises de recentrage du Sujet (comme "Peuple", "Histoire", "Raison", etc.) qui se jouait encore dans l'esthétique eisensteinienne. Et dès lors, le cinéma pourra enfin être pensé non plus comme instrument dialectique ou logique de la "relève" d'un "monde" et d'un "sujet " toujours-déjà donnés, mais le révélateur d'un monde et d'un sujet virtuels (à venir). Avec S. M. Eisenstein, l' "automate" permettait d'aller de l'image à la pensée après avoir provoqué le choc ou la vibration d'où devait naître "la pensée dans la pensée" (Deleuze, 1985, p. 216); mais de la pensée à l'image, il y avait encore la figure s'incarnant dans un monologue intérieur "capable de nous redonner le choc" : "automate dialectique" !

Avec Antonin Artaud, c'est à un tout autre régime de pensée que nous assistons ainsi qu'à la mise en jeu d'une dialectique autrement plus complexe: "Ce que le cinéma [désormais] met en avant, ce n'est pas la puissance de la pensée, c'est son "impouvoir" qui ne sera pas conçu comme "simple inhibition" que le cinéma nous apporterait du dehors, mais de cette inhibition centrale, de cet effondrement et de cette pétrification intérieurs, de ce "vol des pensées" dont la pensée ne cesse d'être la victime et l'agent" (Deleuze, 1985, p. 216). Lorsqu'il s'élève à la hauteur de l'automatisme spirituel, le cinéma ne sera plus conçu comme instrument qui sert à re-produire de la pensée ou à représenter du réel, mais comme une machine d'un ordre supérieur qui met la pensée en relation avec un Dehors que l'on ne peut réduire au monde dit "extérieur". Le cinéma comme automate spirituel est cette "machine" qui met la pensée en contact avec un Dehors qui vient subvertir la nature des rapports de représentation qui existent, au cinéma, entre l'image et la réalité. Pour Deleuze, on dira que le cinéma devient producteur d'un composé original d'espace-temps qui métamorphose le réel. Face à ce Dehors que le cinéma nous donne comme une "offrande", la réalité représentée apparaîtra comme une retombée des virtualités cinématographiques.

Pour illustrer cet aspect des choses, je prendrai un exemple spécifique analysé par Deleuze: celui du tout, celui de l'idée de tout au cinéma. Le Tout, nous dit Deleuze, c'est une notion poétique que l'on peut rapporter à Rilke. Mais c'est aussi une 
notion philosophique qui a été élaborée par Bergson. C'est donc aussi un concept "rigoureux et inexact". Mais, comme le précise Deleuze, ce qui est important, c'est de ne pas confondre le Tout, l'idée de tout au cinéma avec l'idée d'ensemble. Et de fait, pour être la réunion d'éléments très divers, un ensemble n'en reste pas moins une structure close, fermée. Il peut renvoyer ou être inclus dans un ensemble plus grand, mais ce dernier ensemble se voudra à son tour clos, et ainsi à l'infini. Mais le Tout, nous dit Deleuze, est d'une tout autre nature; le Tout ne peut être assimilé à un ensemble, parce qu'il "est de l'ordre du Temps " (Bonitzer et Narboni, p. 40). Je cite Deleuze: "[le Tout] traverse tous les ensembles, et c'est lui précisément qui les empêche de réaliser jusqu'au bout leur propre tendance, c'est-à-dire de se fermer complètement" (p. 40).

Reprenant Bergson, Deleuze dira à son tour: "Le Temps, c'est l'ouvert, c'est ce qui change et ne cesse de changer de nature à chaque instant" (p. $\left.40^{13}\right)$. On pourrait traduire cette thèse en disant que le Temps, c'est l'Ouvert, c'est ce qui change toute chose et ne cesse de changer la nature des choses à chaque instant. Le Temps au cinéma, c'est ce Tout, qui n'est pas un ensemble justement, mais le passage perpétuel d'un ensemble à un autre, la transformation d'un ensemble dans un autre. Mon hypothèse de travail devient donc la suivante: Deleuze crée la notion d'automate spirituel pour tenter de penser le rapport temps-tout-ouvert. Deleuze ne dit pas qu'il n'y a que le cinéma qui pense ce rapport; il se contente de dire que "c'est le cinéma qui nous en rend la pensée plus facile". Il y a, en ce sens, pour Deleuze comme une dimension "pédagogique" propre au cinéma. Et de fait, la démonstration est relativement facile lorsqu'on utilise le cinéma comme opérateur d'analyse pour intuitionner un phénomène aussi singulier. Pour "illustrer" son point de vue, Deleuze prend comme "modèle" les trois niveaux d'organisation et d'articulation de l'image suivants: le cadrage, le découpage et le mouvement.

Je cite un extrait d'un entretien donné par Deleuze :

Le cadrage, c'est la détermination d'un ensemble provisoire artificiellement clos [mais qui de toute évidence n'épuise pas ce qui est en jeu dans une image cinémato-

De l'« automate spirituel » ou le temps dans le cinéma moderne selon Gilles Deleuze 
graphique]; mais c'est qu'il est constamment traversé par le découpage qui sera la détermination du ou des mouvements qui se distribuent dans les éléments de l'ensemble; mais le mouvement à son tour exprime aussi un changement ou une variation du tout, qui lui est affaire de montage (p. 40).

Et en ce sens, le Tout sera cette "puissance", cette dunamis qui traverse tous les ensembles et qui les empêche précisément de se fermer "totalement ". Pour illustrer son "cas" d'espèce, Deleuze prend l'exemple de la notion d'espace off qui renvoie, selon Deleuze, à deux réalités distinctes: 1) elle indique d'une part que tout ensemble donné fait partie d'un ensemble plus vaste, à deux ou trois dimensions (il y a les objets qui se trouvent en deçà de la porte et les objets qui sont "hors champ ", audelà. The secret beyond the door!) ; 2) elle indique d'autre part, que tous les ensembles " [...] plongent dans un tout d'une autre nature, d'une quatrième ou cinquième dimension et qui ne cesse de varier à travers les ensembles qu'il traverse, si vastes soient-ils; dans un cas, c'est une extension spatiale et matérielle; mais dans l'autre cas, c'est la détermination spirituelle» (p. 40). Parmi les nombreux "exemples" que Deleuze analyse spécifiquement, il y a bien sûr Dreyer et Bresson dont les films montrent bien combien il est difficile de séparer les deux types de "dimensions": la matérielle et la spirituelle, la cinétique et la temporelle. Chaque cinéaste doit recréer à nouveaux frais son ou ses rapports au Tout qui traversera les ensembles. Mais c'est que, comme le dit Deleuze, dans un grand film, comme dans toute œuvre d'art, "il y a toujours quelque chose d'ouvert" (p. 40). Et si vous cherchez à savoir ce que c'est, vous découvrirez que c'est le temps, c'est le tout, tels qu'ils sont produits par l'" automate". Car l'automate n'est autre que cette "machine" qui permet d'ajouter $n$ dimensions à la première et la deuxième dimension de l'image: "champ contrechamp".

Voici donc l'idée de Tout arrachée à celle de totalité organique ou dialectique. Mais cela n'est pas tout justement, car c'est encore grâce à la mise en place de l'idée du "T.-A.S. " que Gilles Deleuze mettra à jour les différences qui existent entre un régime "théorématique ${ }^{14}$ " et un régime "problématique" de la 
pensée cinéma et sera amené à repenser totalement le rapport du cinéma à son "langage" et à ses "objets". En effet, muni du "T.-A.S.", Deleuze pourra aisément montrer que le cinéma d'Eisenstein ou de Godard par exemple ne renvoie pas seulement à une différence d'idéologie ou d'esthétique, mais surtout à une différence préalable de position à l'égard de la nature des "problèmes" que pose le cinéma et de la manière de les résoudre. Ainsi, d'un cinéaste à l'autre, d'une esthétique à l'autre, ce ne sont pas seulement deux esthétiques qui s'affrontent ou s'opposent, mais deux nouveaux régimes signifiants en même temps que deux nouveaux régimes de pensée: le régime problématique et le régime théorématique. Deux régimes qui affectent la manière dont on traite du temps et dont le temps nous affecte.

Ce que son analyse nous révèle aussi, et encore une fois cela est intuitionné grâce au "T.-A.S. ", c'est que l'aspect fondamental du "nouveau cinéma" ne doit pas (seulement) être rattaché à telle ou telle particularité d'ordre technique ou historique, mais à un trait "formel " du cinéma qui seul peut permettre de faire la "différence": quelle est la nature du lien qui existe entre l'image-cinéma, ou mieux, la pensée-cinéma et le monde? Or ce que découvre Deleuze, c'est que ce qui les sépare, ce n'est pas tel ou tel différend mondain, mais une " [...] rupture du lien sensorimoteur ("image-action") et plus profondément du lien de l'homme et du monde ("grande composition organique")". Découverte "archéologique" ou, si l'on préfere, "transcendantale" qui "fait la différence", car il ne s'agira plus à présent de négocier les rapports du cinéma au monde en termes psychologiques ou pratiques, mais de dégager un nouveau champ de pensée: un champ et une situation "psychiques" purs, et les modes d'agencement spécifiques d' "op-signes" et de "sonsignes" que les cinéastes devront mobiliser pour être à même de faire face à une vision qui les emporte dans un indéfini comme un devenir trop puissant pour eux: l'impensable ${ }^{15}$ ! "C'est trop beau! Trop dur à supporter!" (Rossellini). "Impossible à dire! Å formuler!» (Godard). Cinéma de voyant.

Dans de telles conditions, et tel est ici l'apport du "T.-A.S.", ce qui caractérisera l'œuvre d'un Eisenstein à ce niveau, ce sera 
moins tel ou tel "tic" formel, tel ou tel "idéologème", que la prévalence qu'il accorde à une pensée où ce seront «les rapports intérieurs de principe à conséquences" (Deleuze, 1985, p. 227) qui prévaudront. Par contre, ce qui caractérisera l'œuvre d'un Godard ou d'un Pasolini, ce sera le caractère "problématique" de la rencontre des images et des sons, des "op-signes" et des "sons-signes" avec le "monde" qui sera privilégié, en faisant "[...] intervenir [cette fois] un événement du dehors, ablation, adjonction, section, qui constitue ses propres conditions et détermine [...] les "cas”" (p. 227). Mais c'est que le cinéma n'est plus conçu comme un "appareil" ou un "dispositif" qui nous permet de représenter le monde, de manière plus ou moins réaliste ou adéquate, mais comme un "automate" - et c'est en cela qu'il est dit "spirituel" — qui nous permet plutôt de "connaître notre propre puissance de connaître" (p. 227). Ce qui découle de ces prémisses, c'est que selon l'orientation - le "choix" - que l'on fait, ce n'est pas du tout aux mêmes "figures" ou "types" de temporalité que l'on aboutit. Aux "types" eisensteiniens viendra non pas forcément s'opposer, mais s'ajouter, une multiplicité de "figures" auxquelles correspondra une variété de "croyances" nouvelles: l' "inévocable» wellesien, l' "inexplicable" robbegrilletien, l' "indécidable" resnaisien, l' "impossible» durassien, ou encore l' incommensurable" godardien; chacune accompagnée de sa propre sensibilité au temps (p. 237). Autant de variations qu'il aurait été impossible de mettre à jour - de lever, comme aime bien à le dire Deleuze sans le scanning qu'a rendu possible le "T.-A.S. " et qui à présent apparaissent comme des formes ou des retombées de la puissance noétique intrinsèque du cinéma comme "automate spirituel "!

Ce que, grâce à son opérateur, Gilles Deleuze sera à même de montrer par la suite, c'est qu'à l'exigence théorique de dégager un nouveau statut de l'image du cinéma moderne correspond la nécessité de remodeler ou de réévaluer la rhétorique qui avait prévalu dans la théorie du cinéma avant la découverte du "T.-A.S». En effet, ce que Deleuze découvre en même temps qu'il arrache l'image cinématographique au champ "narratifreprésentatif " qui la tenait prisonnière d'une pensée abstraite ou simplement "logique", c'est qu'on ne peut avoir intuitionné ce 
qui lie le cinéma au dehors de toute représentation sans faire que justement la "logique" qui présidait à la production de ses objets demeure "inentamée ".

Si le premier aspect de la révolution qui affecte le cinéma était la rupture du "lien sensori-moteur" et celui de l'"homme et du monde", le "[...] deuxième aspect [de cette rupture] sera le renoncement aux figures, métonymie non moins que métaphore, et plus profondément la dislocation du monologue intérieur comme matière signalétique du cinéma" (Deleuze, 1985, p. 226). Plus de métaphores, plus de métonymies et moins encore de "monologues intérieurs", non point parce que le cinéma serait devenu amorphe ou indifférencié ou encore insensible à la "poésie", mais plus profondément "[...] parce que la nécessité propre aux rapports de pensée dans l'image a remplacé la contiguïté des rapports d'images (champ contrechamp)" (p. 226). Par exemple, le champ contrechamp du cinéma "classique" sera désormais remplacé par la profondeur de champ, la plongée ou la contreplongée de Welles ou d'Astruc, le "modèle" de Bresson ou les "coupures irrationnelles" de J.-L. Godard ou de Pasolini, soit par des "figures hyperspatiales" (p. 228) qui ne renvoient plus les images cinématographiques à la représentation prétendument "familière" du monde, mais " [...] à un dehors qui fait passer [les images] les unes dans les autres, comme des projections coniques ou des métamorphoses" (p. 228). Quand la perception devient optique et sonorité pures, ce n'est plus avec le simple mouvement ou l'action qu'elle entre en rapport, mais avec une image virtuelle, "image mentale ou en miroir", dit Deleuze. C'est le nouvel "automatisme" qui fera que l'image actuelle et l'image virtuelle (se) mêleront pour produire l' "image-cristal ", toujours double, toujours redoublée telle qu'on la trouve chez Renoir, Ophüls, Fellini. Comme l'a bien montré Maurizio Grande dans la belle étude qu'il a consacrée au travail de Deleuze sur le cinéma, "Le temps au miroir": "L'image-cristal est une sorte de temps-au-miroir. En elle l'actuel est toujours un présent, mais le présent est l'image actuelle avec en plus son propre passé rendu contemporain." Comme "automate spirituel ", le cinéma nous donnera à expérimenter une image qui ne sera plus "seulement coupée de la chose et du corps", "pas 
seulement autre chose par rapport à la représentation, au souvenir, et à la reconnaissance qui maintiennent une relation physique et mentale avec les schèmes sensori-moteurs de l'action", mais une image — "hallucinatoire", si l'on veut — "saisie dans l'instant même de sa dissolution, dans l'instant de sa cristallisation en une temporalité immédiate, dirigée et sans articulation avec quelque chose d'autre par rapport à l'image qui se forme dans le cristal-temps" (p. 61). C'est dire que seul l'"automatisme" sera à même de nous permettre de penser l'apport du cinéma à notre appréhension du temps comme "décrochage des images du corps et de la réalité", soit "le temps en tant qu'image immédiate sans corps possible, qui se traduit comme Voyance et comme Hallucination" (p. 61) Le mode de cristallisation change à chaque fois, mais ce que l'on expérimente à chaque fois, c'est une nouvelle qualité du Temps et de son "nappé" (Barthes). C'est dans ce "décrochage" par rapport au corps et à la conscience - comme "aperception originairement synthétique" d'un Je-Moi-présent-à-soi-même - que Deleuze situe la révolution qui a affecté le cinéma moderne en son essence. Mais c'est que dès lors qu'il s'est élevé à la puissance de l'"automatisme spirituel ", le cinéma renverse le rapport du sujet à son corps en particulier à sa "vision" — et à sa mémoire, car " [...] l'image ne dérive plus d'un corps percevant ni d'un cerveauarchives de données, ou dormant ou mémorisant [...] ; elle conduit le sujet à la dérive trans-perceptive et "falsifiante", l'aspirant dans son monde" (p. 60).

Devenu "automate spirituel" ("machine" non dialectique), le cinéma devra aussi conquérir une nouvelle force: celle qui en fera un "automate" toujours, mais cette fois, comme machine a-grammaticale et a-rhétorique. Une machine qui aura le pouvoir de " $[\ldots]$ porter l'image au point où elle devient déductive et automatique, [et de] se substituer aux enchaînements représentatifs ou figuratifs sensori-moteurs" (Deleuze, 1985, p. 226227). Pour Deleuze, le cinéma moderne est (devenu) "automate spirituel " désormais en ce qu'il est à même de faire du déroulement du film un véritable théorème et non plus simplement une pure association d'images et ainsi "rendre la pensée immanente à l'image" (p. 226). C'est cette "immanence», produit direct du 
nouvel "automatisme" qui traverse le cinéma, qui permet seule de bien comprendre la véritable "mutation" qui viendra affecter les autres concepts du cinéma et en changer l'économie.

La profondeur de champ, par exemple, ne sera plus située par rapport "à des obstacles ou à des choses cachées" - conception encore toute "mécanique" qui la réduit à une prouesse technique ou à un simple trait esthétique — « [...] mais par rapport à une lumière qui nous fait voir les êtres et les objets en fonction de leur opacité" (Deleuze, 1985, p. 230). Parce qu'elle fait partie intégrante d'une totalité "ouverte", elle a aussi " [...] un effet mental de théorème, elle fait du déroulement du film un théorème et non une association d'images, elle rend la pensée immanente à l'image" (p. 226). De même, le plan-séquence ne sera plus seulement un instrument, parmi d'autres, de la production d'une image-action dominée par le mouvement et le déplacement des objets et des êtres dans un espace neutre, mais "élément " ou " point " d'un problème (p. 229). C'est encore grâce au "T.-A.S." que les notions de hors-champ et de faux raccord se verront accorder leur nouveau statut par rapport à un "tout " qui, lui aussi, subira une mutation, "[...] parce qu'il a cessé d'être l'Un-Être, pour devenir le "et" constitutif des choses, l'entre-deux constitutif des images" (p. 235). Dorénavant, l'automatisme matériel des images et des sons ne renverra plus à un monde donné ou à une intériorité psychologique préformée, mais provoquera du dehors - un dehors qui n'est plus homogène à l'extériorité - "une pensée qu'il impose, comme l'impensable à notre automatisme intellectuel" (p. 233). Le Tout ne se confondra plus avec une structure plate ou un "modèle", mais avec ce que, en se référant à Blanchot, Deleuze appelle "la force de dispersion du Dehors" ou le vertige de l'espacement: "le cinéma comme générateur d'un nouveau "chaosmos" et comme générateur d'images-cristal mi-actuelles, mi-virtuelles capables de nous redonner à voir un monde qui était lui-même devenu une "hallucination" ".

Ce qu'en fin de compte le "T.-A.S." permet de comprendre mieux, c'est la nature des mutations qui ont conditionné l'irruption d'une image et d'une pensée qui n'ont plus affaire à des choses ou des êtres actuels, mais à un monde optique et sonore

De l' «automate spirituel » ou le temps dans le cinéma moderne selon Gilles Deleuze 
pur qui renvoie à des choses ou des êtres virtuels: soit à un monde à venir. Un monde où l'image actuelle et l'image virtuelle ont fusionné pour donner naissance à des "cristaux" de temps et d'espace mêlés. En mobilisant la force noétique du "T.-A.S.", ce sont les différents modes de cristallisation du temps que Gilles Deleuze nous a donnés à penser à nouveaux frais pour mieux comprendre et pour mieux voir (par) le cinéma. Or, ce que nous pouvons "voir " grâce au "T.-A.S.", — ou, comme le dit Deleuze encore, grâce à "l'Eil de l'Esprit" — " [...] c'est le Temps, les nappes de temps, une image-temps directe, qui nous rend capables de saisir les mécanismes de la pensée " (Bonitzer et Narboni, p. 38) et par là-même, d'établir de nouveaux liens avec le monde et de croire encore en ce monde-ci.

Mais "croire" ici, ce n'est pas redécouvrir les vertus de la foi, mais " [...] rendre le discours au corps et, pour cela, atteindre le corps avant les discours, avant les mots, avant que les choses soient nommées" (Bonitzer et Narboni, p. 38).

Brown University

\section{NO TES}

1 Nous renvoyons bien sûr aux ouvrages Cinéma I. L'Image-mouvement (Paris: Minuit, 1983) et Cinéma II. L'Image-temps (Paris: Minuit, 1985).

2 Cf. Gilles Deleuze et Félix Guattari dans Qu'est-ce que la philosophie? (Paris: Minuit, 1991) décrivent ainsi le "personnage conceptuel n: "Le personnage conceptuel n'a rien à voir avec une personnification abstraite, un symbole ou une allégorie, car il vit, il insiste. Le philosophe est l'idiosyncrasie de ses personnages conceptuels. C'est le destin du philosophe de devenir son ou ses personnages conceptuels, en même temps que ses personnages deviennent eux-mêmes autre chose que ce qu'ils sont historiquement, mythologiquement ou couramment (le Socrate de Platon, le Dionysos de Nietzsche, l'Idiot de Cuse). Le personnage conceptuel est le devenir ou le sujet d'une philosophie, qui vaut pour le philosophe. [...] " (p.62-63). C'est moi qui souligne.

3 Voir les ouvrages de Deleuze: Spinoza et le problème de l'expression (Paris: Minuit, 1968) et Spinoza (Paris: P.U.F., 1970).

4 Pour suivre ces "nouvelles déterminations", voir Mille Plateaux (Paris: Minuit, 1980).

5 "Quand il montre que nos idées sont causes les unes des autres, [Spinoza] en conclut que toutes ont pour cause notre puissance de comprendre et de penser. Mais surtout, le terme "automate spirituel " témoigne de l'unité. L'âme est une espèce d'automate spirituel, c'est-à-dire: en pensant nous n'obéissons qu'aux lois de la pen-

Cinémas, vol. $5, \mathrm{n}^{\text {o* }} 1-2$ 
sée, lois qui déterminent à la fois la forme et le contenu de l'idée vraie, qui nous font enchaîner les idées d'après leur cause et suivant notre propre puissance, si bien que nous ne connaissons pas notre puissance de comprendre sans connaître par les causes toutes les choses qui tombent sous cette puissance" (Spinoza et le probleme de l'expression, Paris: Minuit, 1968, p. 126). Avec les "déplacements" et les "ajustements" que nous analysons plus haut, Gilles Deleuze dégagera du cinéma un "automatisme" et une "autonomie" de la même force.

$6 \mathrm{Ce}$ "moment" occupe la plus grande partie des analyses du premier livre et se présente comme une relecture de Bergson comme philosophe du mouvement.

7 C'est moi qui souligne.

8 C'est-à-dire, comme nous le verrons plus loin, non dialectique, non grammaticale et non rhétorique pour Deleuze!

9 C'est moi qui souligne.

10 Je fais référence ici à la très belle analyse que Deleuze a proposée du complexe audiovisuel tel qu'il joue dans la pensée de Michel Foucault. Voir Foucault (Paris: Minuit, 1986), notamment le chapitre intitulé "Les plissements ou le dedans de la pensée». Voir aussi, David N. Rodowick, "Reading the Figural", Camera Obscura (Septembre 1990). Rodowick montre bien l'importance des enjeux théoriques que la confrontation entre le régime des "visibilités" et le régime des "énoncés " chez Foucault peut avoir pour la théorie du cinéma : "Rather than closing in on itself, enunciation now obeys a centripetal force derived from the accelerated orbit of the expressible with respect to the increasing density of the visible. The velocity of regimes agitates énoncés like atoms in a particle accelerator. But what new elements-as concepts or possibilities of thought and imagination - will be created? What possibilities of liberation or alienation will they herald (p.33) ? " La conférence d'Edmond Couchot sur l'image "virtuelle" nous a donné une idée adéquate des possibilités nouvelles qui sont offertes au cinéma pour penser le rapport des images cinématographiques au " réel ".

11 Que je transcrirais "T.-A.S. " dorénavant pour mieux rendre compte de sa dimension opératoire et, pour ainsi dire, "machinique": L' "automate spirituel" comme " accélérateur "... de concepts!

12 Soit comme cette "instance démontée, paralysée, pétrifiée, gelée, qui témoigne pour "l'impossibilité de penser qu'est la pensée" (Cinéma 2. L'Image-temps, Paris: Minuit, 1985, p. 217).

13 Dans Cinéma 2. L'Image-temps (Paris: Minuit, 1985), on trouvera cette autre formule: "Le tout précisément ne peut être pensé, parce qu'il est la représentation indirecte du temps qui découle du mouvement" (p. 205). C'est moi qui souligne.

14 Pour une mise au point théorique sur ces deux régimes, $c f$. Gilles Deleuze, Cinéma 2. L'Image-temps (Paris: Minuit, 1985), p. 226 seq et ce qui suit : "Le problématique se distingue du théorématique (ou le constructivisme de l'axiomatique) en ce que le théorème développe des rapports de principes à conséquences, tandis que le problème fait intervenir un événement du dehors, ablation, adjonction, section, qui constituent ses propres conditions et détermine le "cas", ou les cas" (p. 227).

15 "C'est l'automatisme matériel des images, écrit Deleuze, qui fait surgir du dehors une pensée qu'il impose, comme l'impensable à notre automatisme intellectueln (1985, p. 233).

\section{OUVRAGES CITÉS}

Bonitzer, Pascal et Narboni, Jean. "La photographie est déjà dans les choses. Entretien avec Gilles Deleuze ". Cahiers du Cinéma, n 352 (octobre 1983). 
Colombat, André Pierre. "A Thousand Trails: to Work with Deleuze". A Review of Literary Criticism, n 66 (1991).

Deleuze, Gilles. Cinéma 2. L'Image-temps. Paris: Minuit, 1985.

Deleuze, Gilles. Spinoza et le problème de l'expression. Paris : Minuit, 1968.

Deleuze, Gilles et Guattari, Félix. Qu'est-ce que la philosophie? Paris: Minuit, 1991.

Grande, Maurizio. "Le temps au miroir", dans Roberto di Gaetano, Deleuze. Penser le cinéma. Rome: Bulzoni, 1992.

Rodowick, David N. "Reading the Figural ». Camera Obscura (septembre 1990).

Spinoza, B. de. Traité de la réforme de l'entendement. Texte, traduction et notes par A. Koyré. Paris: Joseph Vrin, 1969 et 1985. 